\title{
Multistage activity within a diverse set of epi-drugs against Plasmodium falciparum parasites
}

Nanika Coetzee ${ }^{1}$, Hilde von Grüning ${ }^{1}$, Mariette van der Watt ${ }^{1}$, Janette Reader ${ }^{1}$, LynMarié Birkholtz ${ }^{1 \#}$

${ }^{1}$ Department of Biochemistry, Genetics and Microbiology, Institute for Sustainable Malaria Control, University of Pretoria, Private Bag x20, Hatfield 0028, South Africa

\# Corresponding author Tel.: +27 12420 2479; fax: +27 12362 5302. E-mail address: 


\section{Abstract}

The epigenome of the malaria parasite, Plasmodium falciparum, is associated with control of various essential processes in the parasite including control of proliferation of asexual development as well as sexual differentiation. The unusual nature of the epigenome has prompted investigations of the potential to target epigenetic modulators with novel chemotypes. Here, we explored the diversity associated with a library of 95 compounds, active against various epigenetic modifiers within cancerous cells, for activity against multiple stages of $P$. falciparum development. We show that $P$. falciparum is differentially susceptible to epigenetic perturbation during asexual and sexual development, with early stage gametocytes particularly sensitive to epi-drugs targeting both histone and non-histone epigenetic modifiers. Moreover, 4 compounds targeting histone acetylation and methylation, show potent multistage activity against asexual parasites, early and late stage gametocytes, with transmission-blocking potential. Overall, these results warrant further examination of the potential antimalarial properties of these hit compounds. 


\section{Background}

The almost inevitable nature of drug resistance development by malaria parasites enforces continued exploration of novel classes of antimalarial drugs. To contribute to global malaria elimination strategies $(1,2)$, such compounds would need to target multiple life cycle stages including the rapidly dividing asexual parasites for chemotherapies, as well as terminally differentiated sexual gametocytes for transmission-blocking activity. Importantly, to prolong resistance development, new chemical matter targeting novel biological activities in the parasite is needed.

In oncology research, epigenetic therapeutics ('epi-drugs') that inhibit epigenetic modulators evidently hold great promise as targets for anticancer therapies (3). The result is an epigenetic de-regulation with antitumor activity, due to targeting histone modifying enzymes and DNA methyltransferases, or facilitating reader interference by inhibiting or activating epigenetic processes. This results in particularly histone posttranslational modifications (PTMs) and disruption of transcriptional processes, chromatin structure maintenance and DNA repair $(4,5)$. Various anticancer epi-drugs have been approved for clinical use, including Azacitidine, Decitabine, Vorinostat and Romidepsin (6).

Plasmodium falciparum relies heavily on epigenetic mechanisms to drive both asexual proliferation and sexual differentiation (reviewed in (7-11)). The parasite's genome encodes a unique complement of histone modifying enzymes including histone deacetylases (HDACs), histone acetyltransferases (HATs), histone methyltransferases (HMTs, including lysine HKMT), protein arginine methyltransferases (PRMTs), and histone demethylases (HDMs) (11) in addition to other non-histone epigenetic modifiers. As a result, inhibitors of particularly histone modifying enzymes have been investigated as novel chemotypes in antimalarial drug discovery efforts (12-22).

Selective anticancer epi-drugs have been investigated for their activity against $P$. falciparum asexual parasites and to a lesser extent, against gametocyte stages. These compounds disturb the parasite's gene expression, ultimately leading to cell death (2022). HDACs are seen as particularly promising drug targets due to resultant 
hyperacetylation (on various histone sites) upon disruption of these activities, with HDACi (HDAC inhibitors) receiving the vast majority of interest. This includes wellknown hydroxymate-based inhibitors like SAHA (suberoylanilide hydroxamic acid, Vorinostat and its derivates) and TSA (Trichostatin A) as well as cyclic tetrapeptides like apicidin, which have shown selective inhibition against asexual $P$. falciparum stages $(17,22-26)$ and gametocytes $(17,26)$. SAHA additionally retained activity in clinical isolates of both $P$. falciparum and $P$. vivax (27). These data have led to larger screens for diverse and selective inhibitors of HDACs $(14,28-30)$. In $P$. falciparum, histone lysine methyltransferases (HKMTs) are involved in both transcriptional activation (through $\mathrm{H} 3 \mathrm{~K} 4$ me marking) and repression (e.g. H3K9me marks), and are also hypothesised to be promising drug targets (16), with BIX01294 (as model HKMT inhibitor, HKMTi) successfully inhibiting asexual $P$. falciparum proliferation and gametocyte viability $(15,16)$. The diaminoquinazoline chemotype has shown to be particularly effective HKMTi against asexual $P$. falciparum parasites, with diversity set screens resulting in selective inhibitors identified $(15,16,19)$. Although these data support the notion that epigenetic modulators could be drug targets in parasite development as well as differentiation, some chemotypes show overt toxicity, poor selectivity and sometimes poor pharmacokinetics (31). Diverse chemotypes targeting various epigenetic modulators should therefore be explored.

In this study, a library of anticancer compounds (Cayman Epigenetics Screening Library, Cayman's Chemicals, USA) with known capabilities to inhibit diverse epigenetic modulators in cancerous cells, was evaluated for their antiplasmodial activity against multiple $P$. falciparum stages. The library consists of 39\% HDACi and 15\% HKMTi; with the remaining compounds divided into 11 other inhibitor subtypes including targeting of HAT, DNA demethylases (DNDM), DNA methyltransferases (DNMT), protein arginine deiminases, PRMT, bromodomain proteins, HDMs, lysinespecific demethylases (LSD), and processes involved in hydroxylation and phosphorylation. As the unusual epigenome and associated regulatory machinery of the parasite provide extensive biology to be investigated, the use of this diverse library of epi-drugs could prioritise which epigenetic modifiers have potential as novel druggable entities. This study describes a comprehensive screening of inhibitors of epigenetic modulators against multiple life cycle stages of $P$. falciparum, including asexual parasites, early (immature) and mature late stage gametocytes and gamete 
formation. We demonstrate that $\mathrm{HDACi}$ and $\mathrm{HKMTi}$ remains the most potent compounds with multistage activity but identify new chemotypes with the potential to be used as chemical starting points for antimalarial drug discovery efforts.

\section{Results}

\section{Comparative profiling of the Cayman Epigenetics library for inhibition activity} against $\boldsymbol{P}$. falciparum parasites

All 95 compounds in the Cayman Epigenetics library were profiled for in vitro activity against asexual and sexual $P$. falciparum parasites in a dual-concentration screen (1 and $5 \mu \mathrm{M}$, Figure $1 \mathrm{~A}$, supplemental figure 1, supplementary file 1). This included stage-specific evaluation of the compounds against early (>85\% stage II/III) and late stage (>95\% stage IV/V) gametocytes. The majority of the compounds ( $76 \%$ against asexual parasites, early (69\%) and late (82\%) stage gametocytes) showed no/minimal activity. Although similar hit rates and compound identities were observed between asexual parasites and early stage gametocytes (24 and 30\% of compounds, respectively, active against these stages at $>50 \%$ inhibition, Pearson correlation $r^{2}$ of $0.5)$, the distribution of compounds displaying moderate activity against early stage gametocytes were almost double that against asexual parasites (18 vs. 10\%). Overall, the compounds were the least active against late stage gametocytes ( $18 \%$ hit rate). Additionally, the nature of the compounds active against asexual parasites and late gametocytes (and between those active against early and late stage gametocytes) showed poor correlation ( $\mathrm{r}^{2}$ of 0.3 and 0.2 , respectively), indicating some stagespecificity in the distribution of the compounds active against each stage.

Hierarchical clustering of the compounds based on Euclidean distances further revealed this stage-specific distribution (Figure 1A). A subset of 17 compounds (including well known compounds like TSA, SAHA, BIX01294 and HC Toxin), display activity against all life cycle stages tested, marking these compounds as multistage inhibitors. However, 4 compounds (PCI34051, C646, 3-amino benzamide and sirtinol) show late stage gametocyte preference with an additional 10 compounds clustered due to increased activity towards early stage gametocytes. 
The multistage activity of the 95 compounds was stratified based on the inhibitor classes descriptors for these compounds (Figure 1B). As expected, the majority of the active compounds are classified as HDACi and $\mathrm{HKMTi}$, and compounds from these classes target all life cycle stages (Figure 1C). Interestingly, compounds classified in the library as potential DNDM, DNMT, PRMT and HAT inhibitors were within the 'hit' pool. However, inhibitors targeting bromodomain proteins, hydroxylation, phosphorylation, and demethylation activities (both histone demethylase and lysinespecific demethylase) were not particularly active ( $<50 \%$ inhibition). Compounds with $>50 \%$ inhibition (at $5 \mu \mathrm{M}$ ) against all three parasite stages included mostly HDACi (Scriptaid, HC Toxin, ITF 2357, Tenovin-6, CAY10603, M 344, Oxamflatin, Pyroxamide, Trichostatin A, CAY10398, SAHA, Chidamide) and some HKMTi (Chaetocin, UNC0638, BIX01294) (Figure 2C). Within these compounds, some showed distinct dual stage-specific activity against asexual and early gametocyte stages (CBHA, Ellagic Acid, SB939, Suberohydroxamic acid, 3-Deazaneplanocin A, (-)-Neplanocin A, 4-iodo-SAHA), or both gametocyte stages (UNC1999) (Figure 1C). An additional subset of compounds is solely active against asexual stages and early stage gametocytes and include DNDMi (gemcitabine), PRMTi (ellagic acid). Stageselective activity was identified for sinefungin, targeting only asexual parasites or early stage gametocytes (S-Adenosylhomocysteine, 5-Azacytidine, GSK 343, Nicotinamide, JGB1741, Zebularine, CCG-100602) or late stage gametocytes (3amino Benzamide, C646, Sirtinol, PCI 34051) being targeted.

\section{Antiproliferative activity against asexual parasites}

Of the hits described above, 19 compounds were selected for $\mathrm{IC}_{50}$ determination against both drug sensitive (3D7) and resistant (K1 and W2) strains of $P$. falciparum parasites (Figure 2A, Table 1). Collectively, $\mathrm{IC}_{50}$ values ranged between $7 \mathrm{nM}$ to $6 \mu \mathrm{M}$ for all the parasite strains evaluated. The resistance indices $\left(\mathrm{RI}\right.$; ratio of the $\mathrm{IC}_{50}$ value of the resistant strain to the sensitive strain, i.e. K1/3D7 and W2/3D7) averaged at 1.12, indicating limited cross-resistance to the $\mathrm{K} 1$ and $\mathrm{W} 2 P$. falciparum parasite strains (Table 1). Five compounds were extremely potent at $<100 \mathrm{nM}$ against all parasite strains, with the most active compounds (3-Deazaneplanocin A, BIX01294, UNC0638) classified as HKMTi, followed by two HDACi, HC Toxin and TSA. TSA, 
BIX01294 and SAHA showed similar IC 50 values to those found in previous reports $(16,17,22)$. 3-Deazaneplanocin A could, however, consistently not cause complete parasite clearance, even up to $100 \mu \mathrm{M}$ or prolonged exposure (up to $96 \mathrm{~h}$ tested). All of the compounds with activity below $500 \mathrm{nM}$ belong to the HDACi class, emphasizing the importance of this activity for asexual proliferation of $P$. falciparum.

Since the compounds in the library were included based on evidence of activity against mammalian lines, their selective toxicity towards $P$. falciparum parasites was determined (Table 1). All of the most potent compounds, except for HC Toxin, showed preference towards $P$. falciparum parasites, with particularly BIX01294 and UNC0638 highly selective towards the parasite with $S \mid>300$, supporting that HKMT activity is essentially important to malaria parasite proliferation. CAY10603 and suberohydroxamic acid (HDACi) and ellagic acid (a PRMTi), were also 10-fold more active against the parasite than mammalian cells with $\mathrm{SI}$ values $>10$. These compounds therefore result in a base set of chemical entities that can be explored in medicinal chemistry programs, with selective inhibition towards malaria parasites.

\section{Gametocytocidal and gametocidal activity}

Selected compounds were evaluated for their activity on both early and late stage gametocytes, with 8 compounds showing activity at $<5 \mu \mathrm{M}$ on gametocytes (Figure 3 ). Comparative low $\mu \mathrm{M}$ activity was observed against both early and late stage gametocytes for the HKMTi Chaetocin ( $\mathrm{IC}_{50}: 0.92 \pm 0.29 \mu \mathrm{M}$ early gametocyte; $1.34 \pm$ $0.17 \mu \mathrm{M}$ late gametocyte) and for $5 \mathrm{HDACi}$ :CAY10603 (IC $\mathrm{I0}_{50}: 1.6 \pm 0.8 \mu \mathrm{M}$ vs $1.30 \pm$ $0.29 \mu \mathrm{M}$ early vs late gametocyte), ITF 2357 (IC $50: 3.0 \pm 0.9 \mu \mathrm{M}$ early gametocyte $\mathrm{IC}_{50}$; $2.23 \pm 0.09 \mu \mathrm{M}$ late gametocyte), Oxamflatin (1.2 $\pm 0.6 \mu \mathrm{M}$ early gametocyte $\mathrm{IC}_{50} ; 3.0$ $\pm 0.4 \mu \mathrm{M}$ late gametocyte $\left.\mathrm{IC}_{50}\right), \mathrm{HC}$ Toxin $\left(2.817 \pm 0.269 \mu \mathrm{M}\right.$ early gametocyte $\mathrm{IC}_{50}$; $2.25 \pm 0.54 \mu \mathrm{M}$ late gametocyte $\mathrm{IC}_{50}$ ). The HDACi Scriptaid had marginal preference towards late stage gametocytes $\left(3.16 \pm 0.625 \mu \mathrm{M}\right.$ early gametocyte $\mathrm{IC}_{50} ; 1.096 \pm 0.02$ $\mu \mathrm{M}$ late gametocyte $\mathrm{IC}_{50}$ ), similarly to sirtinol with potent activity against these more mature gametocytes $\left(0.112 \pm 0.015 \mu \mathrm{M}\right.$ late gametocyte $\left.\mathrm{IC}_{50}\right)$. Activities for known compounds SAHA, BIX01294 and TSA were comparative to reported values (SAHA: $\left(1.41 \pm 0.13 \mu \mathrm{M}\right.$ early gametocyte $\mathrm{IC}_{50} ; 0.81 \pm 0.21 \mu \mathrm{M}$ late gametocyte $\mathrm{IC}_{50}(17)$; BIX01294: $0.014 \pm 0.002 \mu \mathrm{M}$ early gametocyte $\mathrm{IC}_{50} ; 5.86 \mu \mathrm{M}$ late gametocyte $\mathrm{IC}_{50}$ 
(15); TSA: $0.09 \pm 0.01 \mu \mathrm{M}$ early gametocyte $\mathrm{IC}_{50} ; 4.67 \pm 1.09 \mu \mathrm{M}$ late gametocyte $\mathrm{IC}_{50}$ $(17))$.

CAY10603, Chaetocin and Oxflamfatin was able to reduce the normal $\sim 3: 1$ ratio of female:male mature gametocytes to equal proportions (Figure 3B). In addition, Chaetocin and sirtinol, and to a lesser extent CAY10603, affected the functional viability of male gametocytes to exflagellate ( $>60 \%$ inhibition) (Figure $3 \mathrm{C}$ ). With these also affecting female gametocytes, it points to shared biological activities being targeted rather than sex-specific processes.

\section{HDACi and HKMTi as multistage targeting epi-drugs}

As the HDACi and HKMTi showed the most extensive activity against multiple $P$. falciparum parasite stages, the potential for structure activity relationships between these two inhibitor classes were explored (Figure 4). Although the majority $(>80 \%)$ of the compounds were not structurally related, even though they did show activity in the primary screen against multiple stages, a core hydroxamate-based scaffold could be identified for $5 \mathrm{HDACi}$, which showed $>80 \%$ structural similarity, including Pyroxamide, SAHA, 4-iodo-SAHA, CAY10433 and Pimelic Diphenylamide 106. Conversely, 4 HKMTi showed $>80 \%$ structural similarity as 4-quinazolinamine-based structures, including BIX01294, UNC0638, UNC0224 and UNC0321. A few other structurally similar pairs were also identified, including CAY10398 and M 344 (both HDACi) that has multistage activity and only differs with a single backbone carbon. These compounds show promise as chemical starting points for antimalarial optimisation as they have unique chemical scaffolds compared to most compounds and have an altered molecular target.

\section{Discussion}

The $P$. falciparum parasite's epigenetic regulatory machinery has previously been shown to be a valuable drug target given the importance of gene expression for parasite development, but remains to be exploited in full $(15,16,22,23,32-35)$. In this study, various compounds, known to target the epigenetic modulators, were 
investigated for their antiplasmodial activity against asexual parasites, early and late stage gametocytes and gametes. Potent chemical scaffolds were identified with multistage activity, and this study again highlights the potential of HDACi and HKMTi to be exploited as lead compounds upon further experimental validation, cytotoxicity reduction and chemical property optimisation.

The majority of the epigenetic inhibitor classes represented in the library did not show any activity against any of the three $P$. falciparum stages tested. Barring compound uptake issues, this suggests that the epigenetic modulators that should have been targeted by compounds in the library are 1) not essential in the survival of the parasite through development, 2) that the homologues for these epi-drugs' mammalian targets are not found in $P$. falciparum or 3 ) cannot be targeted with the compounds within this library. These epigenetic inhibitor classes included phosphorylation, protein arginine deiminase, HDM, hydroxylation, LSD and bromodomain inhibitors. Amongst the nonhistone epigenetic modulators that were targeted, DNDMi, DNMTi and PRMTi were identified. DNMTi were recently identified with in vitro activity against $P$. falciparum and retains oral efficacy in a $P$. berghei mouse model (14). These compounds are selective inhibitors of human DNMT3a (14), with activity only shown against asexual P. falciparum parasites. These DNMTi does not bear any structural relationship with the compounds tested from the Caymans library, where only Zebularine was identified as DNMTi, with selective inhibition against early gametocytes. The DNDMi Gemcitabine was also shown to have stage-specificity for asexual stages, suggesting possible and antagonistic involvement of DNA methylation-linked chromatin condensation in these stages of development (36). PRMTi were moderately active against asexual and early gametocyte stages. This includes Ellagic Acid, which was previously indicated as potent against asexual parasites (37). Although antioxidant pleiotropic effects of this compound cannot be ignored, the multistage activity reported here suggests a possible role of arginine methylation during these stages (38), but not in mature gametocytes.

Histone-associated epigenetic regulators remain overrepresented as targets, both due to the composition of the library screened, but also due to the known importance of histone acetylation and methylation to parasite survival $(21,39)$. However, HDACs and HKMTs remain the activities targeted the most frequently; a single HATi was identified with multistage activity (C 646, specific competitive inhibitor of human p300 
HAT). Hydroxamate-based HDACi and 4-quinazolinamine-based HKMTi remained the most potent chemical scaffolds that target Plasmodium parasite development at the symptomatic asexual and transmissible gametocyte forms. Hydroxamate-based HDACi were previously shown to have potent antiplasmodial activity with limited cytotoxicity, and this class contains some clinically approved compounds which have been attempted in drug repurposing studies $(22,24,40-43)$. These compounds lead to DNA hyper-acetylation, resulting in the de-regulation of transcription and ultimately cell cycle arrest and cell death $(25,26,44,45)$. Some HDACi were also completely pan-inactive, thereby suggesting that the parasite relies on a specific set of HDACs to regulate its chromosomal condensation via acetylation (reviewed in (46)). The hit HDACi selected in this study display unique chemical scaffolds and showed activity against all stages, although not at equipotent levels (CAY10603, ITF 2357 and Oxamflatin), with potenty increased against asexual parasites and early stage gametocytes. Comparatively, HKMTi overall has the best potency and selectivity with additional activity retained against transmissible stages, similar to previous reports on 4-Quinazolinamine-based HKMTi $(15,16)$. Selective inhibition of HKMT activity can either lead to an increase or decrease in transcription, depending on the position and degree of methylation and ultimately contributes to transcriptional de-regulation and cell death (16). For instance, the HKMTi, 3-Deazaneplanocin A, selectively inhibits H3K27me3 and H4K20me3, and reactivates silenced developmental genes in cancer cells that are not silenced by DNA methylation (47).

The stage-specific inhibition profiles observed for the wide variety of epi-drug inhibitor classes support the findings that the parasite makes use of altered epigenetic regulatory mechanisms to differentiate itself during asexual proliferation and sexual differentiation $(7,48,49)$. Selective Plasmodium inhibition was only shown for 6 compounds of the series, which suggests that the epigenetic modulators targeted by these compounds (HKMT, HDAC and PRMT) show diversity between the parasite and human homologues, as previously shown by the unique set of Plasmodium-specific epigenetic factors that differs vastly from those in its mammalian host (39). The primary evaluation of the activity of epi-drugs against the multiple life cycle stages indicated that early stage gametocytes were particularly susceptible to epi-drug inhibition, supported by reports of a unique epigenetic repertoire associated with these stages, where the switch between asexual and sexual stages was accompanied by 
dynamic histone PTM landscape alterations (7). The differentiation between the compounds active against the different life cycle stages correlates with unique and stage-specific histone PTM dynamics during the parasite's life cycle, with clear peaks abundances for some epigenetic marks associated with particular life cycle stages (7, $48,49)$ and highlights the importance of these activities to parasite development.

Collectively, the data implies that the epigenetic modulators affected are essential for parasite development throughout its asexual and sexual life cycle. Our study reveals that certain chemical scaffolds shared between multistage active compounds hold potential as chemical starting points for further development of derivatives with increased potency, selectivity, or improved physico-chemical properties.

\section{Materials \& Methods}

\section{Asexual $P$. falciparum parasite cultivation and antiproliferative assays}

$P$. falciparum parasites were maintained at $37^{\circ} \mathrm{C}$ in human erythrocytes suspended in complete culture medium and ring-stage synchronised as described (50). SYBR Green I fluorescence was used to determine compound activity against asexual ring stages (1\% haematocrit, $1 \%$ parasitaemia), treated with compounds at 1 and $5 \mu \mathrm{M}$ (for primary screening of inhibitory activity), for $96 \mathrm{~h}$ at $37^{\circ} \mathrm{C}$ as described $(50,51)$, chloroquine disulphate $(1 \mu \mathrm{M})$ as positive drug control. SYBR Green I fluorescence was measured using a Fluoroskan Ascent FL microplate fluorometer (Thermo Scientific, excitation at $485 \mathrm{~nm}$ and emission at $538 \mathrm{~nm}$ ). Unless otherwise stated, each compound was screened in technical triplicates for at least three independent biological replicates $(n=3)$. Hit compounds were selected for full dose-response evaluation under the same assay conditions as above, but against drug sensitive (3D7) and drug resistant W2 (chloroquine, quinine, pyrimethamine and cycloguanil resistant) and K1 (chloroquine, pyrimethamine, mefloquine and cycloguanil resistant) $P$. falciparum strains to determine inhibitory concentrations of the compounds needed to affect $50 \%$ of the parasite population $\left(\mathrm{IC}_{50}\right)$. Data are representative of at least 3 biological repeats, performed in technical triplicates. Assay performances were evaluated with average $\% \mathrm{CV}$ at 4.47 and Z-factors at $>0.6$. 


\section{Parasite lactate dehydrogenase assay to determine inhibition against the early and late stage $P$. falciparum gametocytes}

Gametocytes were induced from asexual $P$. falciparum NF54 parasites as described before (50). The parasite lactate dehydrogenase (pLDH) assay was performed as previously described $(50,52,53)$. Early and late stage $P$. falciparum gametocyte cultures (2\% haematocrit, $5 \%$ gametocytaemia) were treated with compounds at 1 and $5 \mu \mathrm{M}$ (primary dual-point primary screening), with methylene blue $(5 \mu \mathrm{M})$ as positive control for inhibition. Gametocytes were treated under drug pressure for $72 \mathrm{~h}$ at $37^{\circ} \mathrm{C}$, followed by drug washout with culture medium and remaining pLDH activity was determined $72 \mathrm{~h}$ later by addition of Malstat reagent (53) and absorbance measured with a Multiskan Ascent 354 multiplate scanner (Thermo Labsystems, Finland) at 620 $\mathrm{nm}$. A full dose-response evaluation was performed for hit compounds against early and late stage $P$. falciparum gametocytes for three independent biological replicates. Assay performances were evaluated with average \%CV at 4.54 and Z-factors at $>0.5$.

\section{Data analysis}

Chemi-informatics evaluation of compound activities including structure-activity landscape analysis was performed with Osiris DataWarrier v4.7.2

\section{Gamete formation assays.}

Male and female gametocytes were detected visually with Giemsa stain after $48 \mathrm{~h}$ drug pressure, by counting at least 1000 cells. The male exflagellation inhibition assay (EIA) was performed by capturing movement of exflagellation centres over time by video microscopy (adapted from Ghosh et al., 2010). Mature gametocyte culture (>95\% stage $\mathrm{V} ; 1 \mathrm{ml}$ ) was centrifuged at $3500 \mathrm{rpm}$ for 30 seconds and the pellet resuspended in $30 \mu \mathrm{l}$ of ookinete medium (RPMI-1640 medium containing L-glutamine (SIGMA, R6504), $0.024 \mathrm{mg} / \mathrm{ml}$ gentamycin (HyClone, SV30080.01), $202 \mu \mathrm{M}$ hypoxanthine (SIGMA, H9636), 25 mM HEPES (SIGMA, H4034), 0.2\% Glucose (SIGMA, G6152), 0.5\% (w/v) Albumax II (Invitrogen, Paisley, UK) and supplemented with $20 \%$ human serum). The activated culture $(10 \mu \mathrm{l})$ was introduced into a Neubauer chamber and placed on the microscope platform to settle homogenously. Time was noted as time zero $\left(T_{0}\right)$ and the chamber incubated at room temperature (RT). Movement was recorded by video microscopy using a Carl Zeiss NT 6V/10W Stab microscope, fitted with a MicroCapture camera at 10X magnification and then 
quantified by a semi-automated method, a modification of the method described by (54). A series of 15 videos of 8-10 seconds each were captured at random locations between minute 15 and 22.5 after incubation. Each video was analysed using lcy bioimage analysis software in order to quantify the number of exflagellating centres.

\section{Author Contributions}

LMB conceived the study. NC, HvG, MvdW, JR conducted experiments, all authors interpreted results. NC, LMB wrote the paper with inputs from the other authors. All co-authors approved the final version of the paper.

\section{Acknowledgements}

The UP ISMC acknowledges the South African Medical Research Council (SA MRC) as Collaborating Centre for Malaria Research. This work was supported by the South African Research Chairs Initiative of the Department of Science and Technology, administered through the South African National Research Foundation (UID 84627) to LMB.

\section{Competing interests}

The authors declare that they have no competing interests. 


\section{References}

1. Miller LH, Ackerman HC, Su XZ, Wellems TE. Malaria biology and disease pathogenesis: insights for new treatments. Nat Med. 2013;19(2):156-67.

2. Burrows JN, van Huijsduijnen RH, Möhrle JJ, Oeuvray C, Wells TN. Designing the next generation of medicines for malaria control and eradication. Malar $\mathrm{J}$. 2013;6(12):187.

3. Altucci L, Rots MG. Epigenetic drugs: from chemistry via biology to medicine and back. Clin Epigenetics. 2016;8:56.

4. Sun W, Tanaka TQ, Magle CT, Huang W, Southall N, Huang R, et al. Chemical signatures and new drug targets for gametocytocidal drug development. Sci Rep. 2014;4:3743.

5. Dhanak D, Jackson P. Development and classes of epigenetic drugs for cancer. Biochem Biophys Res Commun. 2014;455(1-2):58-69.

6. Audia JE, Campbell RM. Histone Modifications and Cancer. Cold Spring Harb Perspect Biol. 2016;8(4):a019521.

7. Coetzee N, Sidoli S, van Biljon R, Painter H, Llinas M, Garcia BA, et al. Quantitative chromatin proteomics reveals a dynamic histone post-translational modification landscape that defines asexual and sexual Plasmodium falciparum parasites. Sci Rep. 2017;7(1):607.

8. Abel S, Le Roch KG. The role of epigenetics and chromatin structure in transcriptional regulation in malaria parasites. Brief Funct Genomics. 2019.

9. Gupta AP, Bozdech Z. Epigenetic landscapes underlining global patterns of gene expression in the human malaria parasite, Plasmodium falciparum. Int $\mathrm{J}$ Parasitol. 2017;47(7):399-407.

10. Duraisingh MT, Skillman KM. Epigenetic Variation and Regulation in Malaria Parasites. Annu Rev Microbiol. 2018;72:355-75.

11. Cortes A, Deitsch KW. Malaria Epigenetics. Cold Spring Harb Perspect Med. 2017;7(7).

12. Salcedo-Amaya AM, Hoeijmakers WA, Bartfai R, Stunnenberg HG. Malaria: could its unusual epigenome be the weak spot? Int $\mathrm{J}$ Biochem Cell Biol. 2010;42(6):781-4.

13. Trenholme K, Marek L, Duffy S, Pradel G, Fisher G, Hansen FK, et al. Lysine acetylation in sexual stage malaria parasites is a target for antimalarial small molecules. Antimicrob Agents Chemother. 2014;58(7):3666-78.

14. Bouchut A, Rotili D, Pierrot C, Valente S, Lafitte S, Schultz J, et al. Identification of novel quinazoline derivatives as potent antiplasmodial agents. Eur J Med Chem. 2019;161:277-91.

15. Malmquist NA, Moss TA, Mecheri S, Scherf A, Fuchter MJ. Small-molecule histone methyltransferase inhibitors display rapid antimalarial activity against all blood stage forms in Plasmodium falciparum. Proc Natl Acad Sci $U S A$. 2012;109(41):16708-13.

16. Malmquist NA, Sundriyal S, Caron J, Chen P, Witkowski B, Menard D, et al. Histone Methyltransferase Inhibitors Are Orally Bioavailable, Fast-Acting Molecules with Activity against Different Species Causing Malaria in Humans. Antimicrob Agents Chemother. 2015;59(2):950-9. 
17. Trenholme K, Marek L, Duffy S, Pradel G, Fisher G, Hansen FK, et al. Lysine acetylation in sexual stage malaria parasites is a target for antimalarial small molecules. Antimicrob Agents Chemother. 2014;58(7):3666-78.

18. Chua V, Orloff M, Teh JL, Sugase T, Liao C, Purwin TJ, et al. Stromal fibroblast growth factor 2 reduces the efficacy of bromodomain inhibitors in uveal melanoma. EMBO Mol Med. 2019;11(2).

19. Sundriyal S, Chen PB, Lubin AS, Lueg GA, Li F, White AJP, et al. Histone lysine methyltransferase structure activity relationships that allow for segregation of G9a inhibition and anti-Plasmodium activity. Medchemcomm. 2017;8(5):1069-92.

20. Prusty D, Mehra P, Srivastava S, Shivange AV, Gupta A, Roy N, et al. Nicotinamide inhibits Plasmodium falciparum Sir2 activity in vitro and parasite growth. FEMS Microbiol Lett. 2008;282(2):266-72.

21. Andrews KT, Tran TN, Wheatley NC, Fairlie DP. Targeting histone deacetylase inhibitors for anti-malarial therapy. Curr Top Med Chem. 2009;9(3):292-308.

22. Andrews KT, Gupta AP, Tran TN, Fairlie DP, Gobert GN, Bozdech Z. Comparative gene expression profiling of $P$. falciparum malaria parasites exposed to three different histone deacetylase inhibitors. PLoS One. 2012;7(2):e31847.

23. Andrews KT, Haque A, Jones MK. HDAC inhibitors in parasitic diseases. Immunol Cell Biol. 2012;90(1):66-77.

24. Engel JA, Jones AJ, Avery VM, Sumanadasa SD, Ng SS, Fairlie DP, et al. Profiling the anti-protozoal activity of anti-cancer HDAC inhibitors against Plasmodium and Trypanosoma parasites. Int J Parasitol Drugs Drug Resist. 2015;5(3):117-26.

25. Gupta AP, Zhu L, Tripathi J, Kucharski M, Patra A, Bozdech Z. Histone 4 lysine 8 acetylation regulates proliferation and host-pathogen interaction in Plasmodium falciparum. Epigenetics Chromatin. 2017;10(1):40.

26. Ngwa CJ, Kiesow MJ, Papst O, Orchard LM, Filarsky M, Rosinski AN, et al. Transcriptional Profiling Defines Histone Acetylation as a Regulator of Gene Expression during Human-to-Mosquito Transmission of the Malaria Parasite Plasmodium falciparum. Front Cell Infect Microbiol. 2017;7:320.

27. Marfurt J, Chalfein F, Prayoga P, Wabiser F, Kenangalem E, Piera KA, et al. Ex vivo activity of histone deacetylase inhibitors against multidrug-resistant clinical isolates of Plasmodium falciparum and P. vivax. Antimicrob Agents Chemother. 2011;55(3):961-6.

28. Ontoria JM, Paonessa G, Ponzi S, Ferrigno F, Nizi E, Biancofiore I, et al. Discovery of a Selective Series of Inhibitors of Plasmodium falciparum HDACs. ACS Med Chem Lett. 2016;7(5):454-9.

29. Hansen FK, Sumanadasa SD, Stenzel K, Duffy S, Meister S, Marek L, et al. Discovery of HDAC inhibitors with potent activity against multiple malaria parasite life cycle stages. Eur J Med Chem. 2014;82:204-13.

30. Hansen FK, Skinner-Adams TS, Duffy S, Marek L, Sumanadasa SD, Kuna K, et al. Synthesis, antimalarial properties, and SAR studies of alkoxyurea-based HDAC inhibitors. ChemMedChem. 2014;9(3):665-70.

31. Shen S, Kozikowski AP. Why Hydroxamates May Not Be the Best Histone Deacetylase Inhibitors--What Some May Have Forgotten or Would Rather Forget? ChemMedChem. 2016;11(1):15-21.

32. Cui L, Miao J, Furuya T, Fan Q, Li X, Rathod PK, et al. Histone acetyltransferase inhibitor anacardic acid causes changes in global gene expression during in vitro Plasmodium falciparum development. Eukaryot Cell. 2008;7(7):120010. 
33. Duffy MF, Selvarajah SA, Josling GA, Petter M. Epigenetic regulation of the Plasmodium falciparum genome. Brief Funct Genomics. 2014;13(3):203-16.

34. Merrick CJ, Duraisingh MT. Plasmodium falciparum Sir2: an unusual sirtuin with dual histone deacetylase and ADP-ribosyltransferase activity. Eukaryot Cell. 2007;6(11):2081-91.

35. Ukaegbu UE, Zhang X, Heinberg AR, Wele M, Chen Q, Deitsch KW. A Unique Virulence Gene Occupies a Principal Position in Immune Evasion by the Malaria Parasite Plasmodium falciparum. PLoS Genet. 2015;11(5):e1005234.

36. Ponts N, Fu L, Harris EY, Zhang J, Chung DW, Cervantes MC, et al. Genomewide mapping of DNA methylation in the human malaria parasite Plasmodium falciparum. Cell Host Microbe. 2013;14(6):696-706.

37. Soh PM, Witkowski B, Olagnier D, Nicolau M, Garcia-Alvarez M, Berry A, et al. In Vitro and In Vivo Properties of Ellagic Acid in Malaria Treatment. Antimicrobial Agents Chemotherapy. 2009;53(3):1100-6.

38. Zeeshan M, Kaur I, Joy J, Saini E, Paul G, Kaushik A, et al. Proteomic Identification and Analysis of Arginine-Methylated Proteins of Plasmodium falciparum at Asexual Blood Stages. J Proteome Res. 2017;16(2):368-83.

39. Hoeijmakers WA, Stunnenberg HG, Bartfai R. Placing the Plasmodium falciparum epigenome on the map. Trends Parasitol. 2012;28(11):486-95.

40. Chua MJ, Arnold MS, Xu W, Lancelot J, Lamotte S, Spath GF, et al. Effect of clinically approved HDAC inhibitors on Plasmodium, Leishmania and Schistosoma parasite growth. Int J Parasitol Drugs Drug Resist. 2017;7(1):42-50.

41. Dow GS, Chen Y, Andrews KT, Caridha D, Gerena L, Gettayacamin M, et al. Antimalarial activity of phenylthiazolyl-bearing hydroxamate-based histone deacetylase inhibitors. Antimicrob Agents Chemother. 2008;52(10):3467-77.

42. Giannini G, Battistuzzi G, Vignola D. Hydroxamic acid based histone deacetylase inhibitors with confirmed activity against the malaria parasite. Bioorg Med Chem Lett. 2015;25(3):459-61.

43. Sumanadasa SD, Goodman CD, Lucke AJ, Skinner-Adams T, Sahama I, Haque $A$, et al. Antimalarial activity of the anticancer histone deacetylase inhibitor SB939. Antimicrob Agents Chemother. 2012;56(7):3849-56.

44. Horrocks $P$, Wong $E$, Russell K, Emes RD. Control of gene expression in Plasmodium falciparum - ten years on. Mol Biochem Parasitol. 2009;164(1):9-25.

45. Chaal BK, Gupta AP, Wastuwidyaningtyas BD, Luah YH, Bozdech Z. Histone deacetylases play a major role in the transcriptional regulation of the Plasmodium falciparum life cycle. PLoS Pathog. 2010;6(1):e1000737.

46. Hailu GS, Robaa D, Forgione M, Sippl W, Rotili D, Mai A. Lysine Deacetylase Inhibitors in Parasites: Past, Present, and Future Perspectives. J Med Chem. 2017;60(12):4780-804.

47. Miranda TB, Cortez CC, Yoo CB, Liang G, Abe M, Kelly TK, et al. DZNep is a global histone methylation inhibitor that reactivates developmental genes not silenced by DNA methylation. Mol Cancer Ther. 2009;8(6):1579-88.

48. Gupta AP, Chin WH, Zhu L, Mok S, Luah YH, Lim EH, et al. Dynamic epigenetic regulation of gene expression during the life cycle of malaria parasite Plasmodium falciparum. PLoS Pathog. 2013;9(2):e1003170.

49. Karmodiya K, Pradhan SJ, Joshi B, Jangid R, Reddy PC, Galande S. A comprehensive epigenome map of Plasmodium falciparum reveals unique mechanisms of transcriptional regulation and identifies H3K36me2 as a global mark of gene suppression. Epigenetics Chromatin. 2015;8:32. 
50. Reader J, Botha M, Theron A, Lauterbach SB, Rossouw C, Engelbrecht D, et al. Nowhere to hide: interrogating different metabolic parameters of Plasmodium falciparum gametocytes in a transmission blocking drug discovery pipeline towards malaria elimination. Malar J. 2015;14:213.

51. Verlinden BK, Niemand J, Snyman J, Sharma SK, Beattie RJ, Woster PM, et al. Discovery of novel alkylated (bis)urea and (bis)thiourea polyamine analogues with potent antimalarial activities. J Med Chem. 2011;54(19):6624-33.

52. D'Alessandro S, Silvestrini F, Dechering K, Corbett Y, Parapini S, Timmerman $\mathrm{M}$, et al. A Plasmodium falciparum screening assay for anti-gametocyte drugs based on parasite lactate dehydrogenase detection. $J$ Antimicrob Chemother. 2013;68(9):2048-58.

53. Moyo P, Botha ME, Nondaba S, Niemand J, Maharaj VJ, Eloff JN, et al. In vitro inhibition of Plasmodium falciparum early and late stage gametocyte viability by extracts from eight traditionally used South African plant species. J Ethnopharmacol. 2016;185:235-42.

54. Ruecker A, Mathias DK, Straschil U, Churcher TS, Dinglasan RR, Leroy D, et al. A male and female gametocyte functional viability assay to identify biologically relevant malaria transmission-blocking drugs. Antimicrob Agents Chemother. 2014;58(12):7292-302.

55. Hirai S, Endo S, Saito R, Hirose M, Ueno T, Suzuki H, et al. Antitumor effects of a sirtuin inhibitor, tenovin-6, against gastric cancer cells via death receptor 5 upregulation. PLoS One. 2014;9(7):e102831.

56. Kikuchi J, Takashina T, Kinoshita I, Kikuchi E, Shimizu Y, Sakakibara-Konishi $\mathrm{J}$, et al. Epigenetic therapy with 3-deazaneplanocin $\mathrm{A}$, an inhibitor of the histone methyltransferase $E Z H 2$, inhibits growth of non-small cell lung cancer cells. Lung Cancer. 2012;78(2):138-43.

57. Lai YS, Chen JY, Tsai HJ, Chen TY, Hung WC. The SUV39H1 inhibitor chaetocin induces differentiation and shows synergistic cytotoxicity with other epigenetic drugs in acute myeloid leukemia cells. Blood Cancer J. 2015;5:e313.

58. Takai N, Narahara H. Preclinical studies of chemotherapy using histone deacetylase inhibitors in endometrial cancer. Obstet Gynecol Int. 2010;2010:923824. 59. Zappasodi R, Cavane A, lorio MV, Tortoreto M, Guarnotta C, Ruggiero G, et al. Pleiotropic antitumor effects of the pan-HDAC inhibitor ITF2357 against c-Mycoverexpressing human B-cell non-Hodgkin lymphomas. Int $\mathrm{J}$ Cancer. 2014;135(9):2034-45. 
Table 1: Cross-resistant dose-response for the active epi-drugs. Compounds were screened for $\mathrm{IC}_{50}$ against 3D7, K1 and W2 asexual parasite strains using the SYBR Green I-based fluorescence assay. The resistance index (RI; shaded grey) and selectivity index (SI) for each compound is shown. Compounds with $\mathrm{SI}>10$ are shaded in blue. Activity data of the compounds on various mammalian lines were collated from previous reports $(15,23,43,55-59)$. Results for all compounds are representative of three independent biological replicates with technical triplicates $\left(n=3, I C_{50} \pm S E M\right)$.

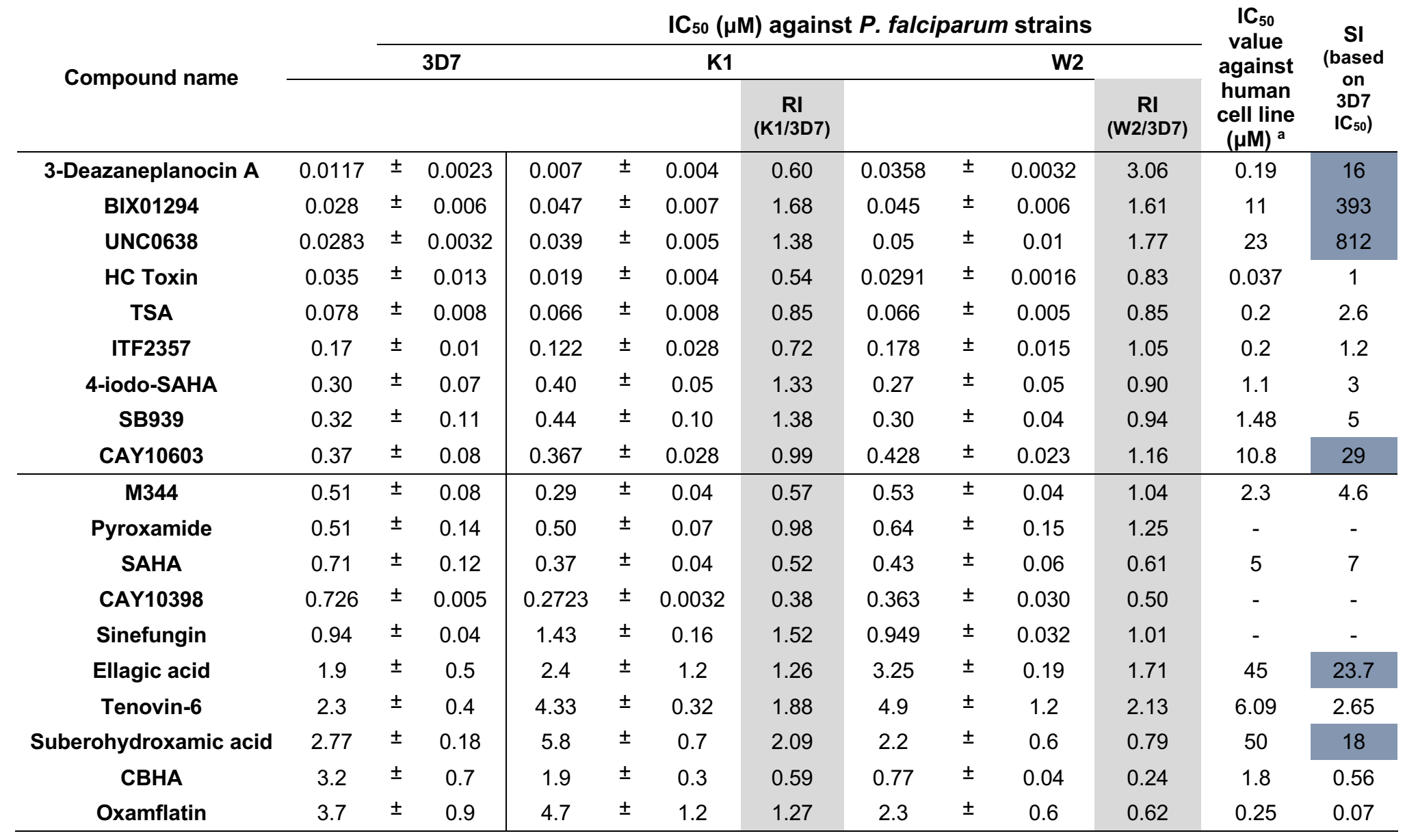




\section{Figure legends}

Figure 1. Comparative profiling of the Cayman Epigenetics library of drugs screened for inhibition activity against $P$. falciparum parasites. (A) Primary compound screening of 95 drugs that inhibit epigenetic modulators was performed using the SYBR Green I-based fluorescence assay for asexual parasites (strains 3D7, $96 \mathrm{~h}$ drug pressure on ring stage parasites) and the pLDH assay for early and late stage gametocytes (strain NF54, $72 \mathrm{~h}$ drug pressure each). The heatmap shows inhibition of asexual parasites and early (EG) and late stage (LG) gametocytes at 1 and $5 \mu \mathrm{M}$ drug pressure. The color scale indicates percentage activity of the treated control after background subtraction of the positive controls (chloroquine for asexual parasites and methylene blue for gametocytes). Compounds with similar inhibition profiles were hierarchically clustered based on Euclidean distance using $\mathrm{R}$ Software (v3.6.0). (B) Distribution of compounds with $>50 \%$ activity per life cycle stage based on their inhibitor classification within the Caymans library. (C) Epi-drug library composition based on inhibitor classification, targeting epigenetic modifiers, with the number of compounds per class indicated. Protein arginine methyltransferase (PRMT), DNA demethylase (DNDM), DNA methyltransferase (DNMT), histone demethylase (HDM), histone lysine methyltransferase (HKMT), lysine-specific demethylase (LSD), histone acetyltransferase (HAT), histone deacetylase (HDAC). Inhibition at $5 \mu \mathrm{M}(\%)$ was compared between asexual parasites (circle size; $n=3$ ) and early $(E G)$ \& late $(L G)$ stage gametocytes $(n=1)$; separated based on the inhibitor type (colour scale corresponding to inhibitor classification as in B). Compounds with multistage activity is identified in the red block and those with asexual and EG preference in the blue block. SHA: suberohydroxamic acid; 3-DC: 3-deazaneplanocin.

Figure 2. In vitro activity of the most active epi-drugs against asexual drug sensitive and resistant $\boldsymbol{P}$. falciparum strains. Compounds were screened using the SYBR Green I-based fluorescence assay to determine dose-response against 3D7 (drug sensitive, black), K1 (drug resistant, red) and W2 (drug resistant, blue). Data are represented as a percentage of untreated control to determine cell proliferation. Sigmoidal dose-response curves were plotted using GraphPad 5.0, from which the $\mathrm{IC}_{50}$ values could be determined. Results for all compounds are representative of three independent biological replicates $(n=3 \pm$ SEM).

Figure 3. Gametocytocidal activity of the selected epi-drugs against early and late stage gametocytes of $\boldsymbol{P}$. falciparum. (A) Compounds were screened using the pLDH assay to determine dose-response against early (>85\% stage II and III, EG) and late $(>95 \%$ stage IV/N, LG) gametocytes after drug pressure for $72 \mathrm{~h}$. Data are represented as a percentage of untreated control. Sigmoidal dose-response curves were plotted using GraphPad 5.0, from which the $\mathrm{IC}_{50}$ values could be determined. Results for all compounds are representative of three independent biological 
replicates ( $n=3 \pm$ SEM), except for sirtinol, scriptaid, HC toxin and UNC0638 $(n=1)$. Where not show, error bars fall within the symbol. Sirtinol was not tested against EG and UNC0638 was not tested against LG. (B) Male:Female gametocyte ratio (males in black bars, females in grey bars) affected by selected compounds. Gametocytes were binned morphologically after evaluating $>1000$ erythrocytes each on Giemsa stained slides. (C)The ability of selected compounds to inhibit male gamete formation. The inhibition of exflagellation of male gametes (\% exflagellating males compared to untreated controls) was visually assessed for two independent biological repeats from 15 videos per repeat, \pm SEM.

Figure 4. Structure activity relationship within the HDAC and HKMT inhibitor series. Structural feature (SkelSphere) analysis was performed with superimposed activity cliff analysis (Osiris DataWarrior V4.2.7) at $80 \%$ structural similarity cut-off. Compounds are limited to HDAC (blue line) and HKMT (red line) inhibitors. Inhibition at $5 \mu \mathrm{M}(\%)$ was compared between asexual parasites (background shading; $n=3$ ), early (EG; circle colour; $n=1$ ) and late (LG; circle size; $n=1)$ stage gametocytes. The active chemical groups shared between two major inhibitor groups are shown; hydroxamate for HDAC and 4-quinazolinamine for HKMT. 
A
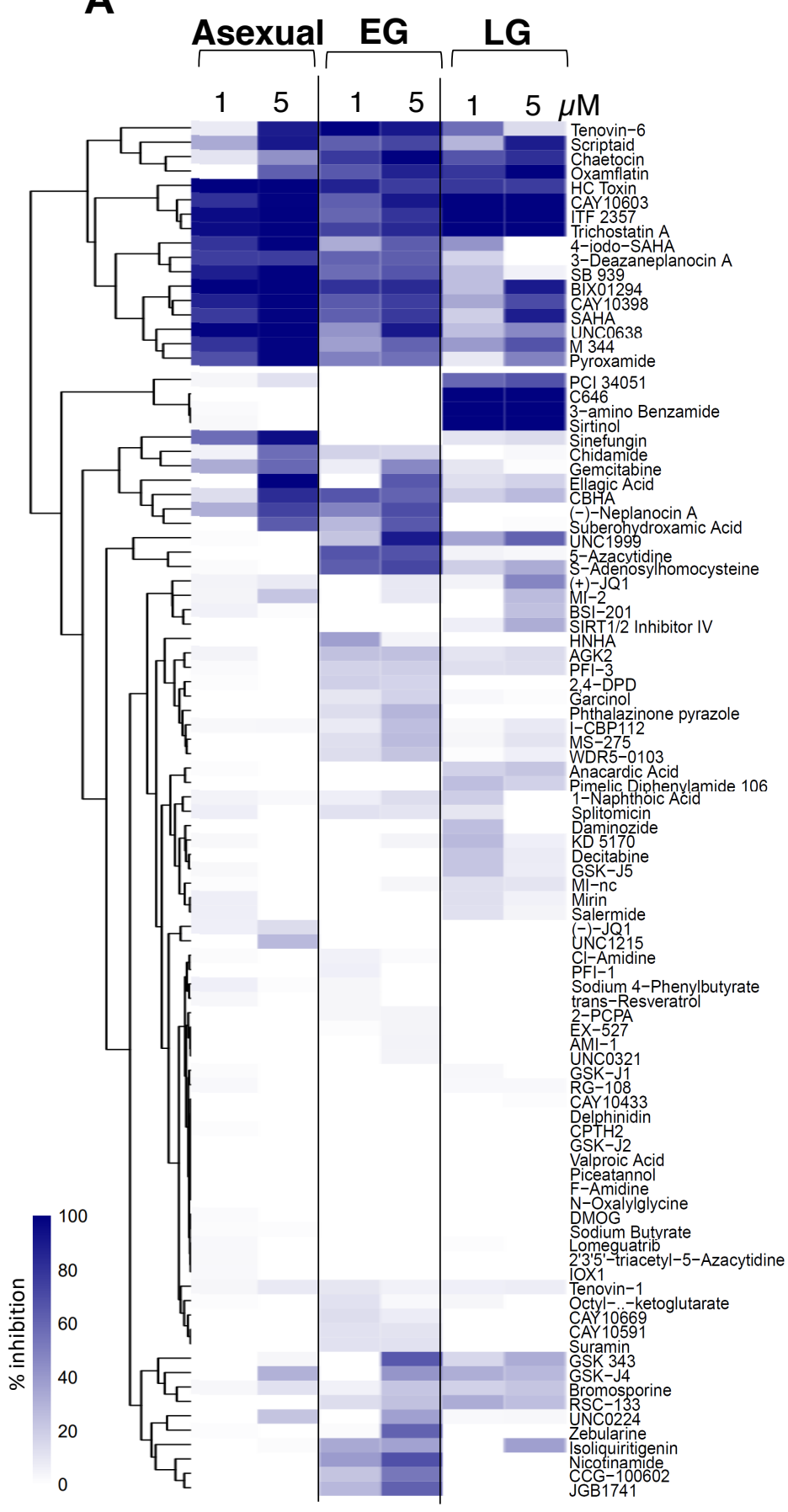

B
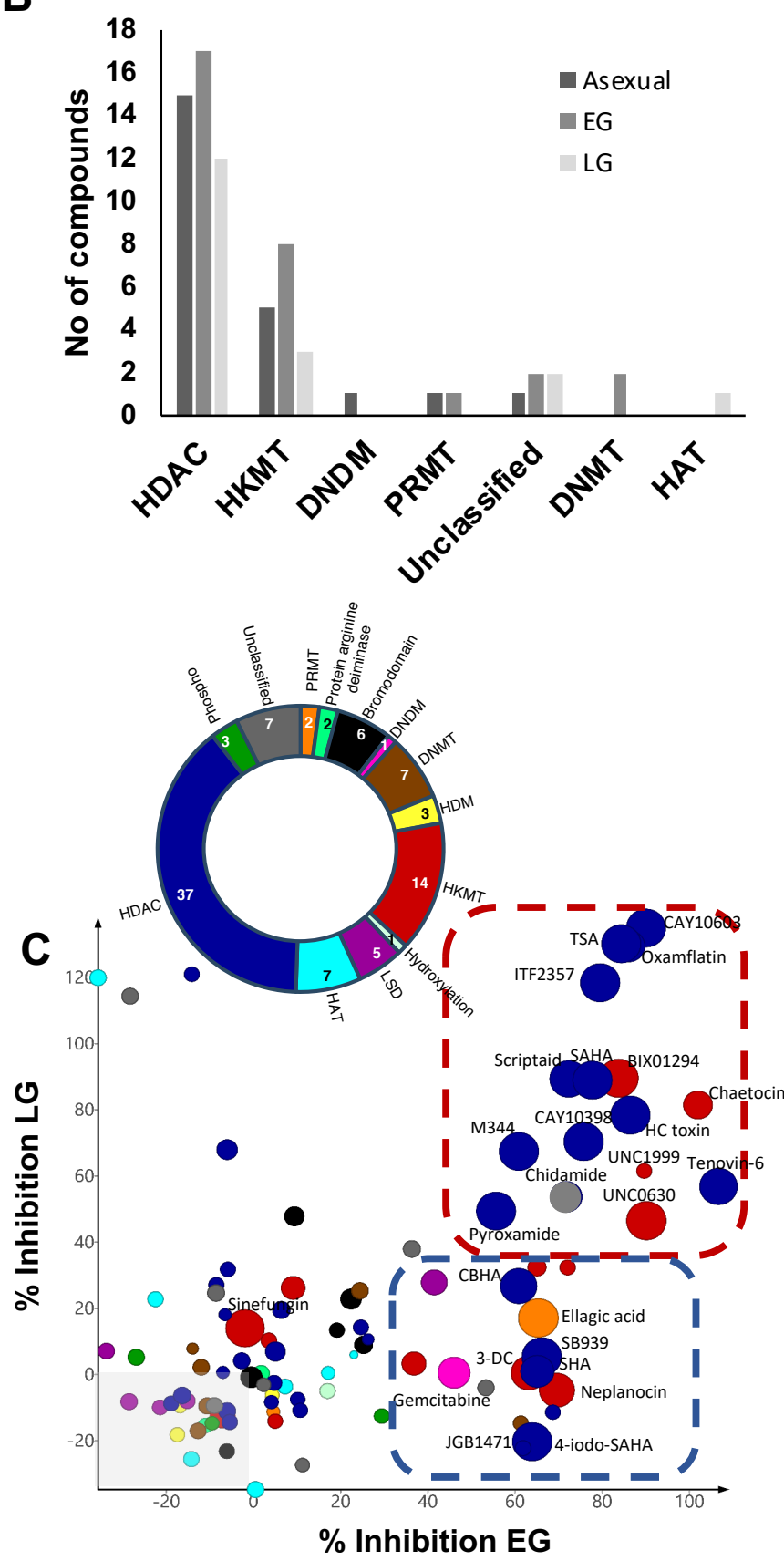

$\begin{array}{cccccc}\text { \% Inhibition } & \bigcirc & \bigcirc & \bigcirc & \bigcirc & \bigcirc \\ \text { Asexual } & 0 & 20 & 40 & \bigcirc & \bigcirc\end{array}$ 


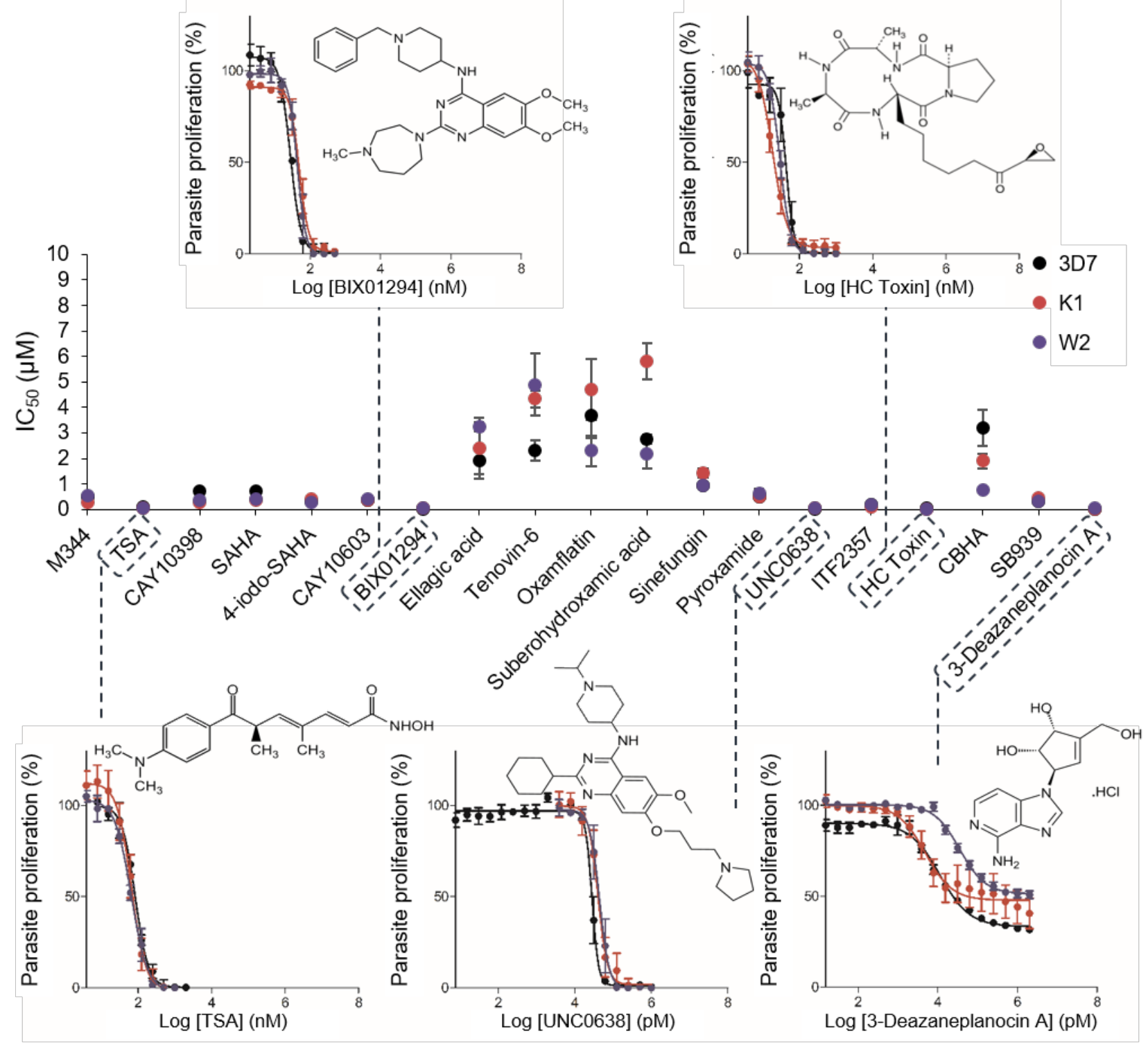




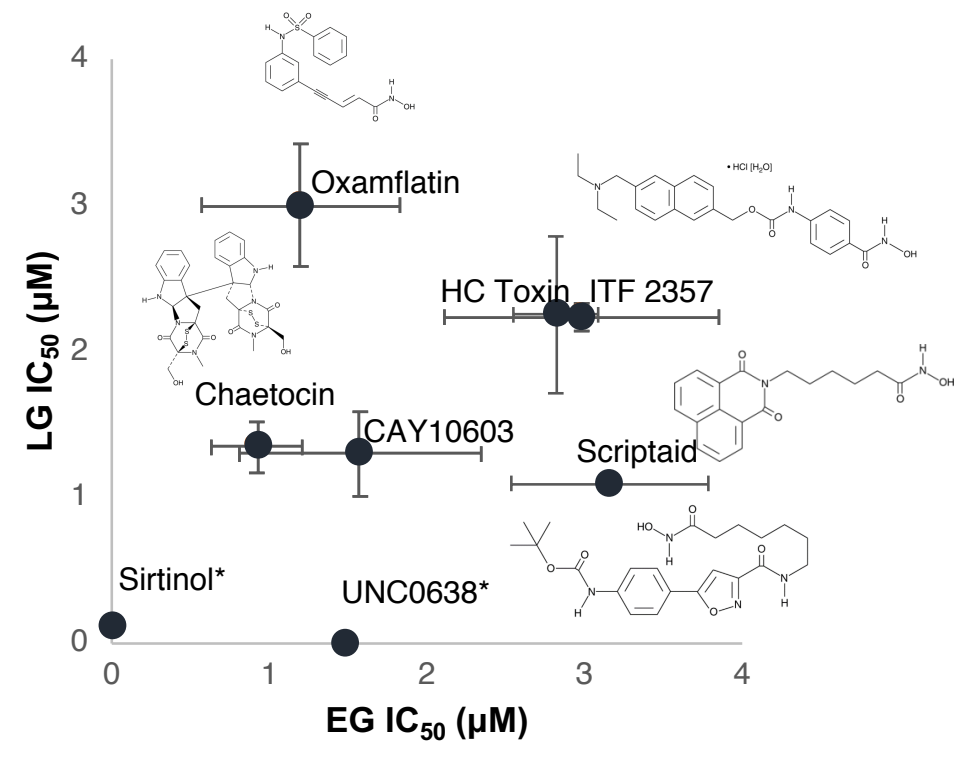

B

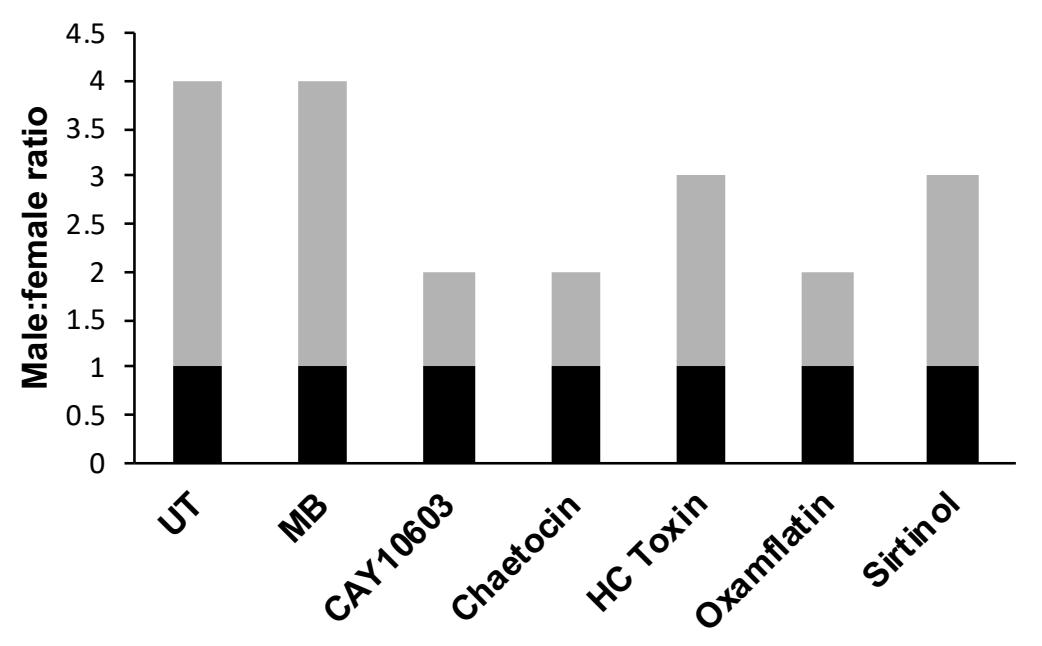

C

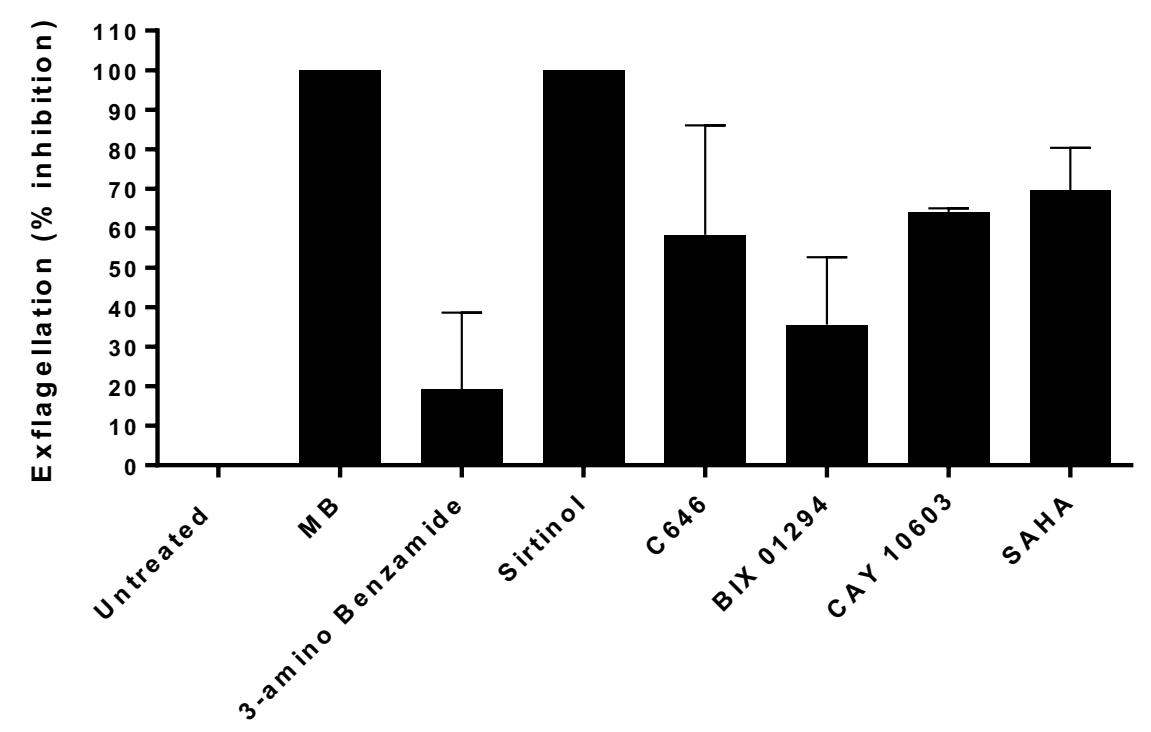



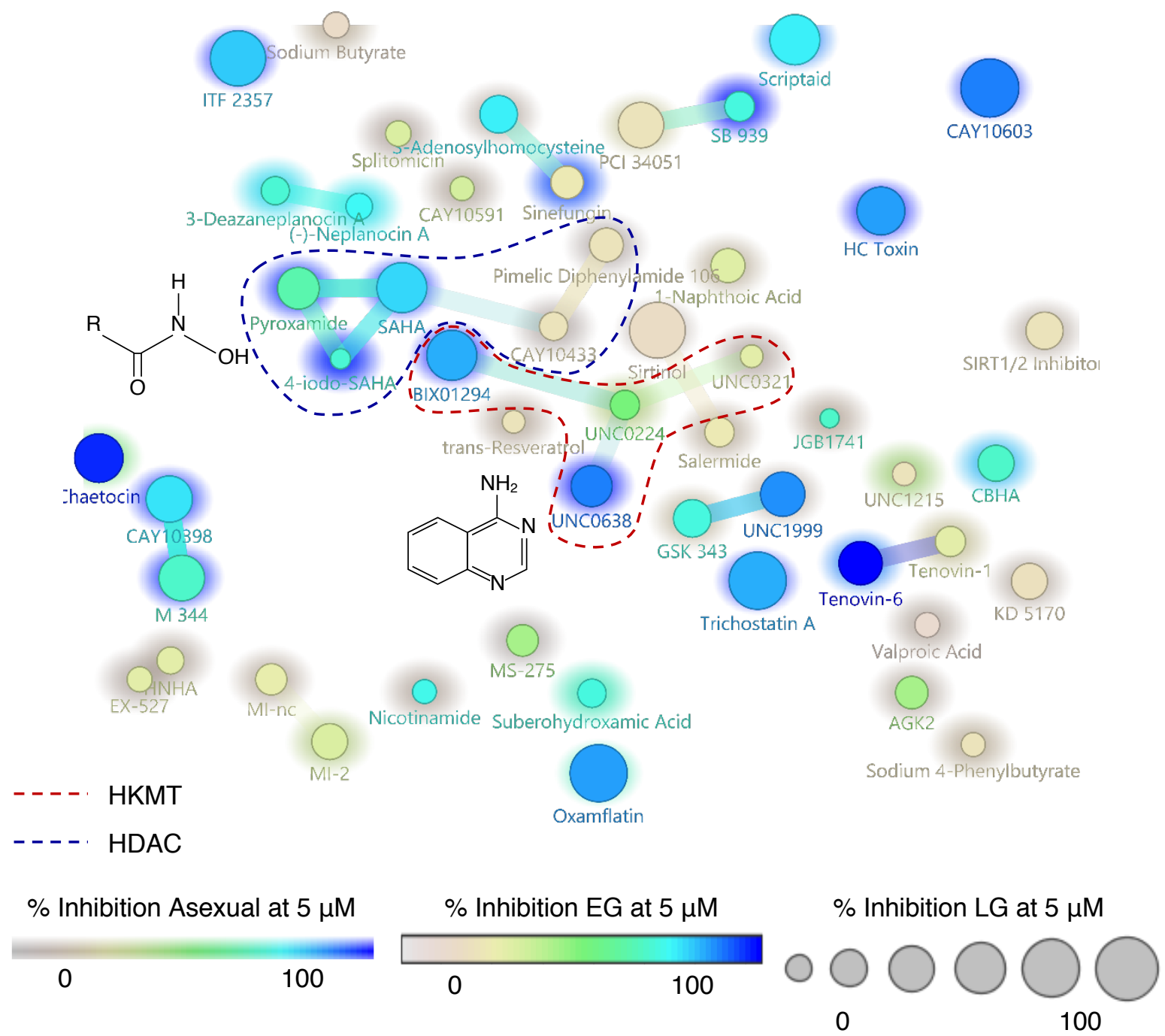